\title{
Research on Seamless Switching Method between Grid and Island
}

\author{
Zhang Mingli, Yu Changyong, Jiang Li, Zhu Heyan, Lu Tianqi, \\ Sun Mingze
}

State Grid Liaoning Electric Power CO, LTD. Power Electric Research Institute, Shenyang, Liaoning110015, China

\begin{abstract}
The seamless switching control strategy between grid-connected microgrid and island operation mode is an important factor to ensure its safe and stable operation. The new master-slave control strategy and the peer-to-peer control strategy are combined to control the switching process of the grid-connected mode of the micro-grid to the island mode. A microgrid simulation model combining photovoltaic cells and batteries was built on the DigSILENT/PowerFactory platform to verify the correctness of the proposed control strategy and ensure the stability of the active, reactive, voltage and frequency of the microgrid.
\end{abstract}

KEYWORDS: microgrid; photovoltaic and battery; PQ control; V/f control; Droop control

\section{Preface}

The microgrid consists of a micro power supply (MS), an energy storage device, an energy management system, and a load. The control of microgrid is an important part of microgrid research [1]. How to realize the seamless switching of microgrid grid-connected and islands is a key issue in microgrid control [2]. The seamless switching control strategy of the microgrid from the grid-connected mode to the island mode can ensure uninterrupted power supply to important sensitive loads and improve the reliability of the user's power supply. Seamless switching means that the microgrid voltage and frequency are within the range specified by the microgrid operating standards during the switching process.

[3-4]. After switching, if the voltage deviation of each busbar in the microgrid is not more than $\pm 7 \%$ of the rated voltage, the frequency deviation is not more than $0.1 \mathrm{~Hz}$, and the microgrid can operate safely and stably. In [5], the inverter adopts multi-loop feedback controller to realize the seamless switching of the microgrid from the grid-connected mode to the island mode. However, it is not studied whether the voltage and frequency after the micro-grid switching remain constant. The basic model of photovoltaic-based microgrid established in [6] has simulated the 
networked operation and island operation state, but has not studied the switching process of microgrid from network to island mode. In this paper, the photovoltaic array and battery model are selected as research objects, which can make the micro-grid grid-connected and island operation, adopt a new integrated control strategy for the battery, and ensure the seamless switching of the micro-grid grid-connected mode to the island mode to ensure the micro-grid is active. Reactive power, voltage and frequency are stable. A microgrid simulation model combining photovoltaic cells and batteries was built on the DigSILENT/PowerFactory platform to verify the feasibility of the proposed control strategy.

\section{Micro power control method}

Most micro-power supplies in the microgrid need to be connected to the grid through a power electronic interface. The basic control methods are constant power control (PQ control), constant voltage constant frequency control (V/f control), and droop control (Droop control).

\subsection{PQ Control}

PQ Control [7 -8] is suitable for MSs with large external influences. The power generation is intermittent. When the microgrid is running in the network, the MS uses PQ control to ensure constant output power.

\subsection{V/f control V/f control}

[9] It is suitable for MS with stable output power. When the capacity is large enough, the system frequency and voltage can be stabilized in the island operation mode.

\subsection{Droop Control}

Droop control [9] is the difference control that can make multiple MSs coordinate coordination. Therefore, it is difficult to realize that the frequency and voltage of the microgrid are equal to those before grid connection, and it is mainly used for microgrid peer control. The active output and reactive output of MS are linear with frequency and voltage.

\section{Battery control method in microgrid}

\subsection{Microgrid grid connection and control strategy of battery during island operation}

At present, the overall control strategy of the microgrid has two modes: 
Academic Journal of Computing \& Information Science

ISSN 2616-5775 Vol. 3, Issue 1: 39-45, DOI: 10.25236/AJCIS.030105

master-slave and peer-to-peer [7]. When the master-slave controlled microgrid is operating in island mode, it has strong laziness to the main control unit, but this control mode can ensure the stability of voltage and frequency. The peer control can not guarantee the stability of voltage and frequency. However, it has the advantages of being simple, reliable, and easy to implement. This paper comprehensively utilizes the advantages of these two control methods to achieve maximum utilization of solar power output. The inverter used in photovoltaic grid-connecting adopts PQ control strategy, and the battery adopts different control modes in different modes, as shown in Figure 1. . When the microgrid is connected to the grid, the battery is controlled by Droop, so that other MSs inside the microgrid do not have to change the previous control mode. When the micro-grid is operating, if $\triangle \mathrm{U} \leq \pm 7 \% \mathrm{U} N$ and $\triangle \mathrm{f} \leq 0$. At $1 \mathrm{~Hz}$, the battery is still controlled by Droop; if $\Delta \mathrm{U}>+7 \% \mathrm{UN}$ or $\Delta \mathrm{U}<-7 \%$ $\mathrm{U} N$ or $\Delta \mathrm{f}>0$. At $1 \mathrm{~Hz}$, the battery is switched to $\mathrm{V} / \mathrm{f}$ control to maintain frequency and voltage stability. The biggest advantage of this control method is that the number of battery switching is reduced, and the probability of successful handover is improved. When the microgrid switches between different operating modes, in order to minimize the amplitude of transient oscillation, a seamless switching control method for tracking the inverter is adopted [10]. The output of the V/f control mode before switching and the output of the Droop control are used as a negative feedback as the input of the V/f control, ensuring that the output of the V/f control is consistent with the output controlled by Droop. When drooping control, K2 and K3 are closed, K1 and K4 are disconnected; when switching, K1 and K4 are closed, and K2 and K3 are disconnected.

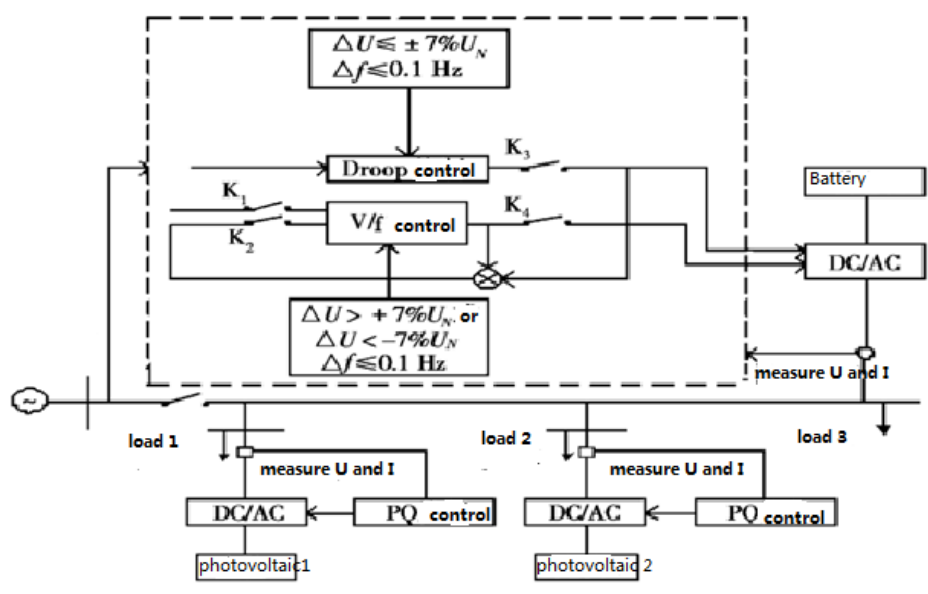

Figure 1 Microgrid control chart

\subsection{Comprehensive control strategy of the battery}

The Droop control and V/f control of the battery are composed of the power outer loop and the current inner loop. Based on the idea of $\alpha \beta / \mathrm{dq}$ conversion and 
power decoupling, the main difference is that the integral link or proportional integral link through the voltage and frequency deviation is different. Therefore, the reference values of active and reactive power are different. The battery integrated control structure designed in this paper is shown in Figure 2, $\triangle \mathrm{U} \leq \pm 7 \% \mathrm{U} N$ and $\triangle \mathrm{f} \leq 0$. At $1 \mathrm{~Hz}$, the battery is controlled by Droop, and the voltage deviation $\Delta \mathrm{U}$ is equal to the voltage reference value $U$ ref and

The difference between the measured values $U \mathrm{~m}$ is obtained by the proportional link, and the reactive power deviation $\Delta \mathrm{Q}$ is obtained; the frequency deviation $\Delta \mathrm{f}$ is equal to the difference between the frequency reference value $f$ ref and the measured value $\mathrm{fm}$, and the active deviations $\Delta \mathrm{P}, \Delta \mathrm{Q}$ and $\Delta \mathrm{P}$ are obtained through the proportional link. Adding the reactive initial value $\mathrm{Q} 0$ and the active initial value $\mathrm{P}$ 0 respectively, and obtaining the active and reactive reference values $P$ ref and $Q$ ref , and then the proportional difference between the active and reactive measured values $\mathrm{P} \mathrm{m}$ and $\mathrm{Q} \mathrm{m}$ After obtaining the current inner loop reference values i dref and $\mathrm{i}$ qref, and then the difference between the abc-dq converted current measurement values id and iq, the switch drive signal $S$ a of the input inverter is obtained by proportional integration and dq/ $\alpha \beta$ conversion, $\mathrm{S} \mathrm{b}, \mathrm{S} \mathrm{c}$, and then realize the grid connection control of the inverter [9].

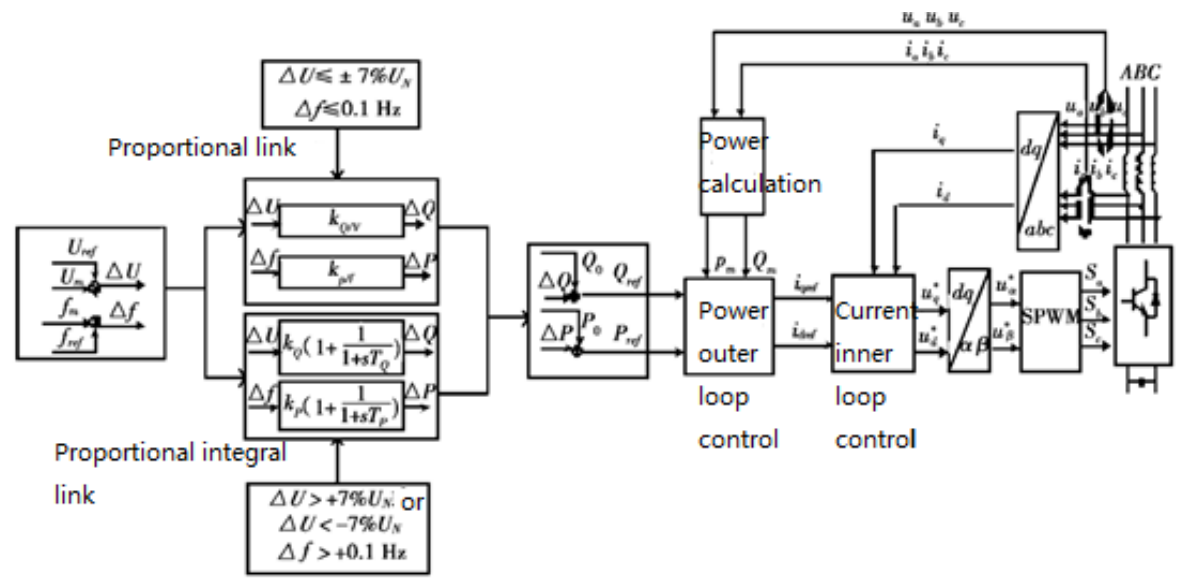

Figure 2 Battery integrated control structure

\section{Simulation analysis}

\subsection{Microgrid model}

The microgrid model adopted in this paper is shown in Figure 3. The $10 \mathrm{kV}$ distribution network is connected to the $400 \mathrm{~V}$ distribution network through the step-down transformer. The distribution network is connected to PV 1 with a capacity of $50 \mathrm{~kW}$; Photovoltaic 2 with a capacity of $100 \mathrm{~kW}$; , capacity $100 \mathrm{~kW} \cdot \mathrm{h}$, 
load 1, load 2 and load 3. This simulation model was built on the DigSILENT/PowerFactory platform. In this paper, the proportional integral constant is determined by the experimental method [9]. When the battery adopts Droop control mode, $\mathrm{K} \mathrm{Q} / \mathrm{V}=12, \mathrm{~K} \mathrm{P} / \mathrm{f}=2$; when using $\mathrm{V} / \mathrm{f}$ control mode, $\mathrm{K} \mathrm{Q}=20, \mathrm{~T} \mathrm{Q}=0$. 3 , $\mathrm{K} \mathrm{P}=1, \mathrm{~T} \mathrm{P}=0$. 5. In the simulation analysis, in order to prevent frequent switching, when the voltage or frequency is $\Delta \mathrm{U}>+7 \% \mathrm{UN}$ or $\triangle \mathrm{U}<-7 \% \mathrm{U}$ or $\Delta \mathrm{f}>0$. When it is within the range of $1 \mathrm{~Hz}$, it needs to be delayed by $0.5 \mathrm{~s}$ switch. 3.2 Simulation Analysis of Microgrid Grid-Connected Mode Switching to Island Mode During micro-grid island operation, when the light intensity and temperature change, if the output of PV 1 and PV 2 is greater than the load demand, the battery absorbs excess reactive power. The simulation results are shown in Figure 4. In Figure $4 \mathrm{a}, \mathrm{t}=0$. At $5 \mathrm{~s}$, the light intensity of Photovolta 2 changes; $t=1$. At $5 \mathrm{~s}$, the temperature of PV 1 changes and the output power does not meet the load requirements. The battery in Figure $4 \mathrm{~b}$ is at $t=0.5 \mathrm{~s}$ and $\mathrm{t}=1$. The active power is increased at $5 \mathrm{~s}$ to maintain power balance. Figure 4c The bus voltage in the microgrid and the system frequency in Figure $4 \mathrm{~d}$ can be stabilized in the battery control mode.

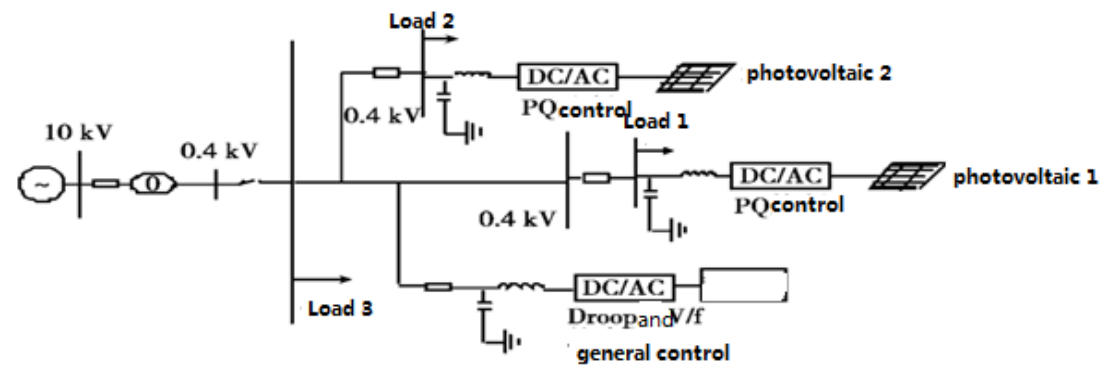

Figure 3 Optical storage hybrid microgrid model

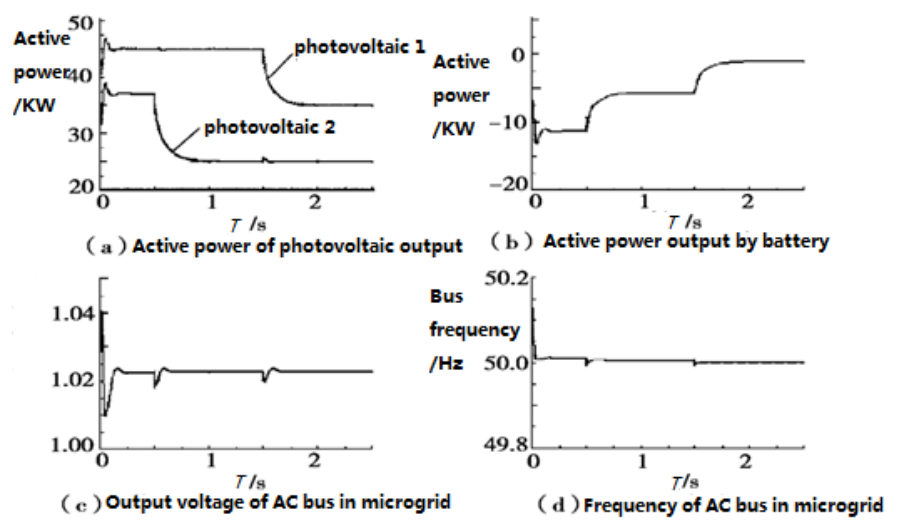

Figure 4 Microgrid output when light intensity and temperature change 
Photovoltaic 1 output $\mathrm{P}=45 \mathrm{~kW}, \mathrm{Q}=0 \mathrm{kVA}$; Photovoltaic 2 output $\mathrm{P}=37 \mathrm{~kW}, \mathrm{Q}$ $=0 \mathrm{kVA}$; load 1, 2, 3 active and reactive power are $45 \mathrm{~kW}$ and $5 \mathrm{kVA}, 47 \mathrm{~kW}$ and 8 $\mathrm{kVA}, 49 \mathrm{~kW}$ and $5 \mathrm{kVA}$. When the microgrid is connected to the grid, the grid provides $70 \mathrm{~kW}$ of active power and $23 \mathrm{kVA}$ of reactive power to meet the load demand. When the microgrid was switched from grid-connected to island mode, PV 1 , PV 2 and load demand did not change. $\mathrm{t}=0$. Before $5 \mathrm{~s}$, the microgrid was networked; $\mathrm{t}=0$. At $5 \mathrm{~s}$, the main network fails, and the microgrid is switched from grid-connected to island mode. The power shortage of the load in the microgrid is provided by the battery. Figure 5 shows a single Droop control for the battery. Figure 6 shows the control strategy combined with Droop control and V/f control. The simulation is compared.

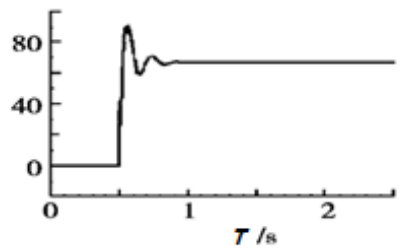

(a) Active power output by battery

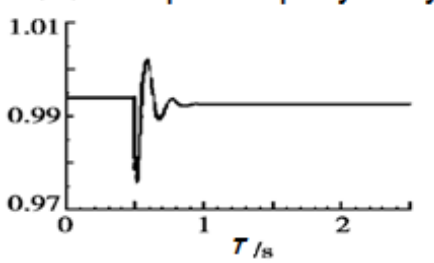

(c) Output voltage of AC bus in microgrid

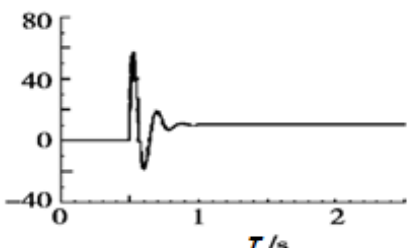

(b) Reactive power output by battery

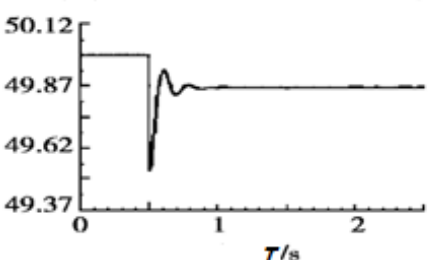

(d) Frequency of $A C$ bus in microgrid

Figure 5 The battery uses the traditional Droop control results

In Figure $5, \mathrm{t}=0$. Before $5 \mathrm{~s}$, when the microgrid is connected to the grid, the battery is controlled by the traditional Droop. The frequency of the system in Figure $5 c$ and the voltage of the busbar in Figure $5 d$ are supported by the large network. The output active and reactive power of the battery in Figure 5a and Figure 5b are zero. $\mathrm{t}=0$. At $5 \mathrm{~s}$, the main network fails, and the microgrid switches to the island operation mode. Figure 5a shows that the battery output active power is increased to $65 \mathrm{~kW}$. Figure 5b shows that the reactive power increase is $18 \mathrm{kVA}$, which meets the requirements of the microgrid internal load. Figure $5 c$ shows the bus voltage value in the microgrid from 0.993 is reduced to 0.991 , the deviation is within the allowable range, and the system frequency is $50.00 \mathrm{~Hz}$ is reduced to 49 . At $87 \mathrm{~Hz}$, the deviation is outside the allowable range and cannot meet the load quality requirements. 


\section{Conclusion}

The seamless switching process of the microgrid grid-connected mode to the island mode is an important factor to ensure the safe and stable operation of the microgrid. In this paper, the new master-slave and peer-to-peer control strategies of the microgrid are used to control the switching process of the microgrid from grid-connected to island operation. The simulation model is established on the DigSILENT/PowerFactory simulation platform to verify the feasibility of the improved control strategy. It ensures that the frequency, voltage and power of the microgrid remain within the allowable range before and after switching, which enhances the ability of the system to operate stably.

\section{References}

[1] Ding Ming, Zhang Yingyuan, Yan Meiqin. Key technologies in microgrid research [J]. Power Grid Technology, 2009, 33(11): 6 -11.

[2] IEEE. 1547. 2-2008 IEEE Standard for Interconnecting Distributed Resources with Electric Power Systems [S]. NewYork: IEEE, 2008.

[3] UL. 1741-2010 Inverters, Converters, Controllers and Interconnection System Equipment for Use With Distributed Energy Resources [S]. Chicago: UL, 2010.

[4] Wang Chengshan, Xiao Zhaoxia, Wang Shouxiang. Multi-loop feedback control strategy for DG inverter in microgrid [J]. Journal of Electrical Engineering, 2009, 24 (2): $100-106$.

[5] Lu Hongyi, Ying Xinlong, He Penteng. Micro-grid networking and island operation control methods [J]. Power System Protection and Control, 2009, 37(11): 28-31.

[6] Zhang Jianhua, Su Ling, Chen Yong, et al. Microgrid energy management and its control strategy [J]. Power Grid Technology, 2011, 35(7): 24 -29.

[7] Zheng Jinghong, Wang Yanting, Li Xingwang, et al. Microgrid smooth switching control method and strategy [J]. Automation of Electric Power Systems, 2011, 35(18): 17 - 24.

[8] Tao Xiaofeng. Research on control strategy of distributed complementary energy microgrid system [D]. Hefei: Hefei University of Technology, 2010.

[9] Li Xuguang. Modeling, simulation and operational characteristics analysis of microgrid [D]. Tianjin: Tianjin University, 2009.

[10] Lu Zongxiang, Wang Caixia, Yong Yong, et al. Summary of microgrid research [J]. Automation of Power Systems, 2007, 31 (19): 100-106. 